\title{
Negative campaigning (Election Campaigning Communication)
}

\section{AUTHOR}

Desiree Steppat, Laia Castro Herrero

\section{KEYWORDS}

negative campaigning, political communication, election campaigns, political ads, negativity, positive ads

\section{BRIEF DESCRIPTION}

One of the most crucial decisions political candidates make ahead of an election is whether they want to focus on their image or that of their their political opponents in their advertisement (Lau and Rovner , 2009). During electoral campaigns, candidates need to decide whether they use political advertisement to display a positive image of themselves or whether they try to make the opponent look bad. The first strategy is referred to as Acclaim or Positive Ads. The second approach, according to Surlin and Gordon is called Negative Campaigning and is applied by a political candidate when (s)he "attacks the other candidate personally, the issues for which the other candidate stands, or the party of the other candidate" (1977, p. 93). However, measuring negative campaigning poses a challenge to academic research since content analyses often fail to address the grey areas of this concept. To begin with, many political ads compare positive characteristics of a candidate against opponents' more negative ones. (Lau \& Rovner, 2009). Ads that contain both strategies, shedding positive light on the candidate while also highlighting negative aspects about the opponent's character or policies are called Comparison or Comparative Ads. These comparisons are difficult to code with straightforward approaches. For example, analyzing campaigns along a positive/negative dichotomy by discounting attacks to the oppo- nent from positive self-presentations may equate strongly positively and negatively charged political advertising to neutral campaigns. Also, negativity in political campaigning is studied in different contexts and has been extended as a number of studies on negative campaigning look in particular at Attacks and Rebuttals/Defense from opponents after an attack (Benoit, 2000; Benoit \& Airne, 2009; Erigha \& Charles, 2012; Lee \& Benoit, 2004; Torres, Hyman, \& Hamilton, 2012). This distinction raises other important methodological and theoretical implications. Sweeping measures of negativity based on common scholarly definitions do not consider voters' tolerance towards the use of certain forms of negativity by candidates (for example, rebutting an attack from an opponent) that may be perceived as legitimate. Not accounting for such nuances is what makes many negativity measures unable to accurately gauge the effects of negative campaigning among the electorate (Sigelman \& Kugler, 2003).

$\overline{\text { FIELD OF APPLICATION/THEORETICAL FOUNDATION }}$ Negative campaigning and its related constructs (such as attacks or rebuttals) have been often associated with current trends in political communication of modernization and professionalization of election campaigns (Voltmer, 2004). Negative campaigning is indeed a development that can be observed across many different political contexts (Kaid \& Holtz-Bacha, 2006). Campaign strategies using negative messages about a political opponent have been studied relying on theories from social and cognitive psychology (Kahn \& Kenney, 1999; Lau, 1985) and mostly in regard to their potential consequences for a healthy democracy (Lau \& Rovner, 2009). Their operationalization follows a simple schema by coding whether a certain construct is present in 
a given advertising piece or not. Alternatively, it is coded which kind of category best reflects on the content of a given political advertisement.

\section{REFERENCES/COMBINATION WITH OTHER METHODS OF DATA COLLECTION}

Negative campaigning and related constructs have been studied through content analysis both of paid advertisement (Benoit, 2000) and news coverage by the mass media (Lau \& Pomper, 2004); The features and effects of negative campaigning have also been analyzed through voter surveys (Brader, 2005, 2006) and interviews with campaign managers (Kahn \& Kenney, 1999). Its effects were furthermore more precisely measured through numerous experimental studies (Ansolabehere, Iyengar, Simon, \& Valentino, 1994; overview see: Lau et al., 2007).

\section{EXAMPLE STUDIES}

see Table 1

\section{REFERENCES}

Ansolabehere, S., Iyengar, S., Simon, A., \& Valentino, N. (1994). Does Attack Advertising Demobilize the Electorate? American Political Science Review, 88(4), 829-838. https://doi.org/10.2307/2082710

Benoit, W. L. (2000). A Functional Analysis of Political Advertising across Media, 1998. Communication Studies, 51(3), 274-295. https://doi.org/10.1080/10510970009388524

Benoit, W. L., \& Airne, D. (2009). Non-Presidential Political Advertising in Campaign 2004. Human Communication, 12(1), 91-117.

Brader, T. (2005). Striking a Responsive Chord: How Political Ads Motivate and Persuade Voters by Appealing to Emotions. American Journal of Political Science, 49(2), 388. https://doi.org/10.2307/3647684

Brader, T. (2006). Campaigning for hearts and minds: How emotional appeals in political ads work. Studies in communication, media, and public opinion. Chicago, Ill.: Univ. of Chicago Press. Retrieved from http://www.loc.gov/catdir/enhancements/fy0622/2005009159-b.html

Buell, E. H., \& Sigelman, L. (2008). Attack politics: Negativity in presidential campaigns since 1960. Studies in government and public policy. Lawrence, Kan.: Univ. Press of Kansas.
Ceccobelli, D. (2018). Not Every Day is Election Day: a Comparative Analysis of Eighteen Election Campaigns on Facebook. Journal of Information Technology \& Politics, 15(2), 122-141. https://doi. org/10.1080/19331681.2018.1449701

Erigha, M., \& Charles, C. Z. (2012). Other, Uppity Obama: A Content Analysis of Race Appeals in the 2008 U.S. Presidential Election. Du Bois Review: Social Science Research on Race, 9(2), 439-456. https:// doi.org/10.1017/S1742058X12000264

Geer, J. G. (2010). In defense of negativity: Attack ads in presidential campaigns. Studies in communication, media, and public opinion. Chicago: University of Chicago Press. Retrieved from http://search.ebscohost.com/login.aspx?direct=true\&scope $=$ site \&db=nlebk\&db=nlabk\&AN=319130

Kahn, K. F., \& Kenney, P. J. (1999). Do Negative Campaigns Mobilize or Suppress Turnout? Clarifying the Relationship between Negativity and Participation. American Political Science Review, 93(4), 877-889. https://doi.org/10.2307/2586118

Kaid, L. L., \& Holtz-Bacha, C. (Eds.) (2006). The SAGE handbook of political advertising. Thousand Oaks, Calif.: SAGE Publications.

Kanouse, D. E., \& Hansen, L. R. (1987). Negativity in evaluations. In E. E. Jones, D. E. Kanouse, H. H. Kelley, R. E. Nisbett, S. Valins, \& B. Weiner (Eds.), Attribution: Perceiving the causes of behavior. Hillsdale, N.J.: Erlbaum.

Lau, R. R. (1985). Two explanations for negativity effects in political behavior. American Journal of Political Science. (29), 119-138.

Lau, R. R., \& Pomper, G. M. (2004). Negative campaigning: An analysis of U.S. Senate elections. Campaigning American style. Lanham, Md.: Rowman \& Littlefield.

Lau, R. R., \& Rovner, I. B. (2009). Negative Campaigning. Annual Review of Political Science, 12(1), 285-306. https://doi. org/10.1146/annurev.polisci.10.071905.101448

Lau, R. R., Sigelman, L., \& Rovner, I. B. (2007). The Effects of Negative Political Campaigns: A Meta-Analytic Reassessment. The Journal of Politics, 69(4), 1176-1209. https:// doi.org/10.1111/j.1468-2508.2007.00618.x

Lee, C., \& Benoit, W. L. (2004). A Functional Analysis of Presidential Television Spots: A Comparison of Korean and American Ads. 
https://doi.org/10.1080/01463370409370179

Sigelman, L., \& Kugler, M. (2003). Why Is Research on the Effects of Negative Campaigning So Inconclusive? Understanding Citizens' Perceptions of Negativity. The Journal of Politics, 65(1), 142-160. https:// doi.org/10.1111/1468-2508.t01-1-00007

Steffan, D., \& Venema, N. (2019). Personalised, De-Ideologised and Negative? A Longitudinal Analysis of Campaign Posters for German Bundestag Elections, 1949-2017. European Journal of Communication, 34(3), 267-285. https://doi.org/10.1177/0267323119830052

Surlin, S. H., \& Gordon, T. F. (1977). How
Values Affect Attitudes Toward Direct Reference Political Advertising. Journalism Quarterly, 54(1), 89-98. https:// doi.org/10.1177/107769907705400113

Torres, I. M., Hyman, M. R., \& Hamilton, J. (2012). Candidate-Sponsored TV Ads for the 2004 U.S. Presidential Election: A Content Analysis. Journal of Political Marketing, 11(3), 189-207. https://doi. org/10.1080/15377857.2012.703907

Voltmer, K. (2004). Mass media and political communication in new democracies: Routledge.

Table 1. Overview exemplary studies measuring interaction, discussion, participation, and related

Authors Unit of analysis Constructs Values Reliability

Benoit (2000), Television ads, Acclaim Benoit \& Air- direct mail, newne (2009), Lee spaper ads, and and Benoit candidate web pages (2004)

Acclaims portray the sponsored candidate in a favorable light, both his/her character and/or policy (Benoit, 2000, 281, 295)

$0=$ not present

$1=$ present

Erigha \& Charles

(2012)
Non-negati- A non-negative/advocacy ad ve/ advocacy favors a party's candidate, focusing solely on that individual.
Cohen's kappa

average $=.96$

$1=$ non-negative / advocacy

$2=$ comparison

$3=$ attack ads

(exclusive options)

Torres et al. Presidential can- Non-compa(2012) didate-sponsored rative ad TV ads
If the ad simply mentions positive attributes of a particular candidate without mentioning an opponent, the ad is coded as a non-comparison (positive) ad (p. 196)

$1=$ comparative ad $2=$ negative $\mathrm{ad}$ $3=$ non-comparative ad (exclusive options)
Cohen's kappa average $=.96$
Cohen's kappa average $=.98$ 


\begin{tabular}{|c|c|c|c|c|}
\hline Authors & Unit of analysis & Constructs & Values & Reliability \\
\hline $\begin{array}{l}\text { Steffan \& Ve- } \\
\text { nema (2019) }\end{array}$ & $\begin{array}{l}\text { Campaign pos- } \\
\text { ters }\end{array}$ & $\begin{array}{l}\text { Textual ne- } \\
\text { gative cam- } \\
\text { paigning } \\
\text { Visual nega- } \\
\text { tive cam- } \\
\text { paigning }\end{array}$ & $\begin{array}{l}\text { Based on Lau and Pomper's } \\
\text { (2002), textual/visual negative } \\
\text { campaiging indicates whet- } \\
\text { her the image / text on the } \\
\text { campaign posters referred } \\
\text { to other political parties or } \\
\text { candidates. (p. } 273 \text { ) } \\
0=\text { not present } \\
1=\text { present }\end{array}$ & $\begin{array}{l}\text { Visual nega- } \\
\text { tive campaig- } \\
\text { ning: } \\
\text { Krippendorff's } \\
a=.82 \\
\text { Textual nega- } \\
\text { tive campaig- } \\
\text { ning: } \\
\text { Krippendorff's } \\
a=.84\end{array}$ \\
\hline $\begin{array}{l}\text { Torres et al. } \\
(2012)\end{array}$ & $\begin{array}{l}\text { Presidential can- } \\
\text { didate-sponsored } \\
\text { TV ads }\end{array}$ & Negative ad & $\begin{array}{l}\text { If the ad criticizes the oppo- } \\
\text { sing party and/or candidate } \\
\text { but offers no alternative (in } \\
\text { essence, the ad presents ne- } \\
\text { gative information about an } \\
\text { opponent but no information } \\
\text { about the candidate on whose } \\
\text { behalf it is run), then the ad } \\
\text { is coded as a negative ad. } \\
1 \text { = comparative ad } \\
2 \text { = negative ad } \\
3=\text { non-comparative ad } \\
\text { (exclusive options) }\end{array}$ & $\begin{array}{l}\text { Cohen's kappa } \\
\text { average }=.98\end{array}$ \\
\hline $\begin{array}{l}\text { Ceccobelli } \\
(2018)\end{array}$ & Facebook posts & $\begin{array}{l}\text { Negative } \\
\text { rhetorical } \\
\text { strategy }\end{array}$ & $\begin{array}{l}\text { The posts taken into conside- } \\
\text { ration are those in which lea- } \\
\text { ders employ a purely negative } \\
\text { campaigning strategy. Cases } \\
\text { in which a hypothetic leader } \\
\text { A attacks one or more politi- } \\
\text { cal opponents by comparing } \\
\text { his/her own figure or policy } \\
\text { proposal with the one(s) of } \\
\text { her/his competitor(s) are not } \\
\text { coded, since they denote a } \\
\text { comparative rhetorical strate- } \\
\text { gy (p. 129) } \\
0=\text { not present } \\
1=\text { present }\end{array}$ & $\begin{array}{l}\text { Krippendorff's } \\
\text { a average }=.85\end{array}$ \\
\hline $\begin{array}{l}\text { Benoit (2000), } \\
\text { Benoit \& } \\
(2009), \text { Lee \& } \\
\text { Benoit (2004) }\end{array}$ & $\begin{array}{l}\text { Television spots, } \\
\text { direct mail pie- } \\
\text { ces, newspaper } \\
\text { ads, and }\end{array}$ & Attack & $\begin{array}{l}\text { Portrays the opposing candi- } \\
\text { date in an unfavorable light, } \\
\text { both his/her character and/or } \\
\text { policy (Benoit, 2000, 281, 295) }\end{array}$ & $\begin{array}{l}\text { Cohen's kappa } \\
\text { average }=.96\end{array}$ \\
\hline
\end{tabular}




\begin{tabular}{|c|c|c|c|c|}
\hline Authors & Unit of analysis & Constructs & Values & Reliability \\
\hline & $\begin{array}{l}\text { candidate web } \\
\text { pages }\end{array}$ & & $\begin{array}{l}0=\text { not present } \\
1=\text { present }\end{array}$ & \\
\hline $\begin{array}{l}\text { Erigha \& } \\
\text { Charles } \\
(2012)\end{array}$ & $\begin{array}{l}\text { Television and } \\
\text { web advertise- } \\
\text { ments }\end{array}$ & Attack ads & $\begin{array}{l}\text { Attack ads criticize the } \\
\text { opposing candidate without } \\
\text { referencing the sponsoring } \\
\text { party's candidate (p. 443) } \\
1 \text { = non-negative / advocacy } \\
2=\text { comparison } \\
3=\text { attack ads } \\
\text { (exclusive options) }\end{array}$ & $\begin{array}{l}\text { Cohen's kappa } \\
\text { average }=.96\end{array}$ \\
\hline $\begin{array}{l}\text { Benoit (2000), } \\
\text { Benoit \& } \\
\text { Airne (2009), } \\
\text { Lee \& Benoit } \\
(2004)\end{array}$ & $\begin{array}{l}\text { Television spots, } \\
\text { direct mail pie- } \\
\text { ces, newspaper } \\
\text { ads, and candida- } \\
\text { te web pages }\end{array}$ & Defense & $\begin{array}{l}\text { Defense responds to (refutes) } \\
\text { an attack on the candidate, } \\
\text { both on his/her character } \\
\text { and/or policy (Benoit, } 2000, \\
281,295 \text { ) } \\
0=\text { not present } \\
1=\text { present }\end{array}$ & $\begin{array}{l}\text { Cohen's kappa } \\
\text { average }=.96\end{array}$ \\
\hline $\begin{array}{l}\text { Erigha \& } \\
\text { Charles } \\
(2012)\end{array}$ & $\begin{array}{l}\text { Television and } \\
\text { web advertise- } \\
\text { ments }\end{array}$ & Comparison & $\begin{array}{l}\text { A comparison ad weighs two } \\
\text { credentials, characteristics, } \\
\text { or policystances (p. } 443 \text { ) } \\
1=\text { non-negative / advocacy } \\
2=\text { comparison } \\
3=\text { attack ads } \\
\text { (exclusive options) }\end{array}$ & $\begin{array}{l}\text { Cohen's kappa } \\
\text { average }=.956\end{array}$ \\
\hline $\begin{array}{l}\text { Torres et al. } \\
\text { (2012) }\end{array}$ & $\begin{array}{l}\text { Presidential can- } \\
\text { didate-sponsored } \\
\text { TV ads }\end{array}$ & $\begin{array}{l}\text { Comparative } \\
\text { ad }\end{array}$ & $\begin{array}{l}\text { If the ad criticizes the oppo- } \\
\text { sing party and/or candidate } \\
\text { and recommends alternative } \\
\text { courses of action by compa- } \\
\text { ring two candidates on spe- } \\
\text { cific points so as to present } \\
\text { one in a more positive and } \\
\text { the other in a more negative } \\
\text { light, then the ad is coded as } \\
\text { a comparative ad (p. 195) } \\
1=\text { comparative ad } \\
2 \text { = negative ad } \\
3=\text { non-comparative ad } \\
\text { (exclusive options) }\end{array}$ & $\begin{array}{l}\text { Cohen's kappa } \\
\text { average }=.98\end{array}$ \\
\hline
\end{tabular}

